\title{
Ghost interference with classical partially coherent light pulses
}

\author{
Víctor Torres-Company, ${ }^{1, *}$ Hanna Lajunen, ${ }^{2, \dagger}$ Jesús Lancis, ${ }^{1}$ and Ari T. Friberg ${ }^{3, \dagger}$ \\ ${ }^{1}$ GROC-UJI, Departament de Física, Universitat Jaume I, ES-12071, Castelló, Spain \\ ${ }^{2}$ Optical Instrumentation, VTT Technical Research Centre of Finland, P. O. Box 1100, FI-90571 Oulu, Finland \\ ${ }^{3}$ Department of Microelectronics and Applied Physics, Royal Institute of Technology (KTH), Electrum 229, SE-164 40 Kista, Sweden
}

(Received 7 September 2007; published 8 April 2008)

\begin{abstract}
The two-photon temporal coincidence detection amplitude obeys a pair of equations identical to those of classical partially coherent plane-wave pulses propagating in linearly dispersive media. These equations are also the same as the paraxial Wolf equations, for both the two-photon spatial probability amplitude and the cross-spectral density function. Therefore, a fourfold analogy between space and time, as well as between quantum entanglement and partial coherence, arises. In accordance to this, we predict nonlocal interference structures in a fourth-order interferometric configuration with classical partially coherent pulses under the assumption of Gaussian statistics. As an example, we present the classical temporal counterpart of the ghost diffraction phenomenon. Our work suggests that some time-domain entanglement phenomena that hitherto were considered as uniquely quantum can be mimicked by conventional partially coherent light pulses.
\end{abstract}

DOI: 10.1103/PhysRevA.77.043811

PACS number(s): 42.50.Ar, 42.25.Kb, 42.50.Dv

\section{INTRODUCTION}

Entanglement between particles is one of the most striking features of quantum mechanics. It has led to the resolution of the Einstein-Podolsky-Rosen paradox and to demonstrations of the nonlocal character of quantum correlations. In optics, spontaneous parametric downconversion is the method of choice for producing entangled photon pairs (or biphotons). These photon pairs may be entangled in polarization, momentum, space, frequency, and time [1]. Such photon pairs have been the basic source for experiments in quantum cryptography [2], quantum imaging $[3,4]$, quantum teleportation [5], quantum lithography [6], and quantum optical coherence tomography [7].

In the last few years, quantum imaging and ghost diffraction have been a topic of notable interest $[4,8-11]$. In the earlier experiments $[4,8]$, spatially entangled two-photon states were employed in fourth-order correlation configurations. Later, photon correlation imaging $[9,10]$ and ghost diffraction and interference [11] were achieved with thermal light [12]. In this case, apart from a background level, similar effects as in the quantum case were found $[10,11]$. The mathematical foundation of these two different physical phenomena leading to practically identical results was pointed out in [10]. The analogy relies on the fundamental fact that the two-photon probability amplitude obeys a pair of equations identical to that describing the diffraction of spatially partially coherent light $[13,14]$.

In the temporal domain, analogous ghost diffraction phenomena have recently been reported [15]. These experiments

\footnotetext{
*vtorres@fca.uji.es

${ }^{\dagger}$ Present address: Departamento de Óptica, Universitat de València, ES-46100, Burjassot, Spain

${ }^{\ddagger}$ Also at the Department of Engineering Physics and Mathematics, Helsinki University of Technology (TKK), P. O. Box 3500, FI02015 TKK, Finland and the Department of Physics and Mathematics, University of Joensuu, P. O. Box 111, FI-80101 Joensuu, Finland.
}

consist of spectral coincidence measurements of two-photon pairs in an interferometric arrangement. By spectrally (or temporally) filtering a beam in one arm, various interference structures were obtained. The earlier work of Valencia et al. [16] presents temporal interferometric coincidence measurements with two-photon light. Each photon generated in the pair is focused into a different single-mode fiber placed in each arm of the interferometer. It was shown that the scale of the second-order correlation function of the two-photon temporal probability amplitude could be partially controlled by the fiber dispersion coefficients. More recently, due to applications in quantum-information science, the evolution of two-photon temporal entanglement in first-order dispersive media is gaining increased attention [17].

In this work we demonstrate that results similar to twophoton temporal entanglement can be obtained with classical partially coherent pulses. Specifically, we show that nonlocal time-domain interferences can be achieved with a temporally uncorrelated light source of Gaussian statistics. As an example, a white-noise source is split into two different arms. By placing a temporal modulator in only one arm, we theoretically show that intensity correlation measurements yield a temporal Fraunhofer pattern of the modulation gate function. This result constitutes the classical version of ghost diffraction in the time domain. Our example points out that this previously reported physical phenomenon is not genuinely a quantum feature but it can be emulated with classical sources. More generally, this paper provides additional evidence that quantum effects based on nonlocal correlations can be mimicked with conventional partially coherent sources. Furthermore, we support this claim by showing that the equations that govern the stretching of the temporal probability amplitude of two-photon light in homogeneous, linearly dispersive media are the same as those for partially coherent classical pulses.

\section{FOURFOLD SPACE-TIME ANALOGY}

We start by showing explicitly the fourfold connection between classical and two-photon quantum light in the space 
and time domains. For clarity, we briefly review the main equations developed in [14] and specialize them to a onedimensional case.

Classically, the properties of a non-stationary scalar pulsed light beam are characterized by the second-order mutual coherence function [18] $\Gamma\left(\mathbf{r}_{1}, t_{1} ; \mathbf{r}_{2}, t_{2}\right)$ $=\left\langle E^{*}\left(\mathbf{r}_{1}, t_{1}\right) E\left(\mathbf{r}_{2}, t_{2}\right)\right\rangle$, where $E(\mathbf{r}, t)$ is the complex analytic signal of the fluctuating field at position $\mathbf{r}=(x, z)$ and time $t$ and the angular brackets denote the ensemble average. The coherence properties of the beam can be described, alternatively, in the space-frequency domain [19]. In such a case, we use the two-frequency cross-spectral density function $W\left(\mathbf{r}_{1}, \omega_{1} ; \mathbf{r}_{2}, \omega_{2}\right)$, which is a two-dimensional Fourier transform of $\Gamma\left(\mathbf{r}_{1}, t_{1} ; \mathbf{r}_{2}, t_{2}\right)$. Our aim now is to rewrite the Wolf equations [18] in paraxial approximation. On separating the rapid phase factor as $W\left(\mathbf{r}_{1}, \omega_{1} ; \mathbf{r}_{2}, \omega_{2}\right)$ $=W_{e}\left(\mathbf{r}_{1}, \omega_{1} ; \mathbf{r}_{2}, \omega_{2}\right) \exp \left[-i\left(k_{1} z_{1}-k_{2} z_{2}\right)\right]$, where $k_{j}=\omega_{j} / c$ is the wave number, and invoking the slowly-varying-envelope approximation (SVEA), the equations that govern free-space propagation are

$$
\left[\partial_{x_{j}}^{2}+2 i k_{j}(-1)^{j} \partial_{z_{j}}\right] W_{e}\left(\mathbf{r}_{1}, \omega_{1} ; \mathbf{r}_{2}, \omega_{2}\right)=0,
$$

where $\partial_{a}$ denotes the derivative with respect to the variable $a$ and $j=(1,2)$.

In the quantum regime, for a pure entangled state, the two-photon propability amplitude is $\psi\left(\mathbf{r}_{1}, t_{1} ; \mathbf{r}_{2}, t_{2}\right)$ $=\left\langle 0\left|\hat{E}^{+}\left(\mathbf{r}_{1}, t_{1}\right) \hat{E}^{+}\left(\mathbf{r}_{2}, t_{2}\right)\right| \Psi\right\rangle$. Here $\hat{E}^{+}(\mathbf{r}, t)$ is the positivefrequency part of the optical-field operator at space-time point $(\mathbf{r}, t),|\Psi\rangle$ and $|0\rangle$ represent the two-photon and vacuum states, respectively. The probability of observing one photon at $\left(\mathbf{r}_{1}, t_{1}\right)$ and another one at $\left(\mathbf{r}_{2}, t_{2}\right)$ is proportional to $\left|\psi\left(\mathbf{r}_{1}, t_{1} ; \mathbf{r}_{2}, t_{2}\right)\right|^{2}$. The two-photon probability amplitude may also be expressed in the space-frequency domain, by Fourier transforming $\psi\left(\mathbf{r}_{1}, t_{1} ; \mathbf{r}_{2}, t_{2}\right)$ with respect to $t_{1}$ and $t_{2}$. This leads to a function $\phi\left(\mathbf{r}_{1}, \omega_{1} ; \mathbf{r}_{2}, \omega_{2}\right)$. On factoring out the rapid phases as $\phi\left(\mathbf{r}_{1}, \omega_{1} ; \mathbf{r}_{2}, \omega_{2}\right)$ $=\phi_{e}\left(\mathbf{r}_{1}, \omega_{1} ; \mathbf{r}_{2}, \omega_{2}\right) \exp \left[i\left(k_{1} z_{1}+k_{2} z_{2}\right)\right]$, the function $\phi_{e}$ in the SVEA obeys the propagation equations

$$
\left[\partial_{x_{j}}^{2}+2 i k_{j} \partial_{z_{j}}\right] \phi_{e}\left(\mathbf{r}_{1}, \omega_{1} ; \mathbf{r}_{2}, \omega_{2}\right)=0,
$$

where $j=(1,2)$. Apart from a sign, these equations are identical with Eqs. (1). Obviously, the quantum-optical crossspectral density (for any state) also satisfies Eqs. (1) paraxially. Our result indicates that the aforementioned quantum analogy [14] is mathematically closer to nonstationary (pulsed) light than to stationary beams.

Separability of the two-photon amplitude probability function means absence of entanglement, whereas separability in the mutual coherence function implies full spatial coherence [13]. This analogy between coherence and entanglement has provided the basis to perform coherent imaging in a ghost imaging setup with both quantum and classical sources with the same resolution, though different visibility $[10,11]$. Although this similarity even holds for nonparaxial waves [14], we prefer to restrict ourselves to the paraxial case so as to directly connect the space domain with its temporal counterpart.
Quite recently, in quantum temporal imaging, Tsang and Psaltis [17] demonstrated that the equations that govern the evolution of the temporal entanglement of two-photon states in lossless, homogeneous, first-order dispersive media are mathematically identical to Eqs. (2). More specifically, if $\hat{A}^{+}(z, t)$ is the slowly varying plane-wave envelope operator, the two-photon probability amplitude is $\psi_{e}\left(z_{1}, t_{1} ; z_{2}, t_{2}\right)$ $=\left\langle 0\left|\hat{A}^{+}\left(z_{1}, t_{1}\right) \hat{A}^{+}\left(z_{2}, t_{2}\right)\right| \Psi\right\rangle$, and it satisfies the equations, for $j=(1,2)$,

$$
\left[\partial_{\tau_{j}}^{2}-2 i \frac{1}{\beta_{2}} \partial_{z_{j}}\right] \psi_{e}\left(z_{1}, \tau_{1} ; z_{2}, \tau_{2}\right)=0 .
$$

Here the propagation constant, assumed smooth, is expanded as $\beta(\omega)=\beta_{0}+\beta_{1}\left(\omega-\omega_{0}\right)+\beta_{2}\left(\omega-\omega_{0}\right)^{2} / 2$, with $\beta_{1}$ being the inverse of the group velocity and $\beta_{2}$ the group-velocity dispersion (GVD) coefficient at the central frequency $\omega_{0}$. The times $\tau_{j}$ are measured in a coordinate frame that moves at the speed $\beta_{1}$ of the wave packet, i.e., $\tau_{j}=t_{j}-\beta_{1} z$.

Equations (3) are a quantum-optical version of the wellknown space-time analogy that links paraxial diffraction of 1D beams with first-order temporal dispersion of short light pulses $[20,21]$. This means that many results previously known in one domain can readily be transferred to the other. Importantly, this analogy also exists in the classical context among spatially partially coherent beams and partially coherent plane-wave pulses [22]. Consequently, we point out an analogy overlooked up to now between quantum-mechanical two-photon-state coincidence detection and classical fluctuating optical pulses.

To this end, we consider the mutual coherence function of a conventional, statistically nonstationary, spectrally partially coherent, plane-wave field that propagates in the $z$ direction in a lossless, homogeneous, linearly dispersive medium. This is, for instance, the case of pulse propagation in a singlemode fiber. The slowly-varying-envelope part is, as usual, defined via $\Gamma\left(z_{1}, t_{1} ; z_{2}, t_{2}\right)=\Gamma_{e}\left(z_{1}, t_{1} ; z_{2}, t_{2}\right) \exp \left[-i k_{0}\left(z_{1}-z_{2}\right)\right.$ $\left.+i \omega_{0}\left(t_{1}-t_{2}\right)\right]$, where $k_{0}=\omega_{0} / c$. Under the SVEA, the function $\Gamma_{e}$ satisfies the following equations [22]:

$$
\left[\partial_{\tau_{j}}^{2}-2 i(-1)^{j} \frac{1}{\beta_{2}} \partial_{z_{j}}\right] \Gamma_{e}\left(z_{1}, \tau_{1} ; z_{2}, \tau_{2}\right)=0,
$$

where $j=(1,2)$ and $\tau_{j}$ are the retarded (proper) times. Besides being analogous to Eqs. (1) and (2), these equations are identical (apart from a sign) to those in Eq. (3) for the quantum-optical two-photon temporal probability amplitude.

By the same reasoning as before, a temporally fully incoherent classical pulse corresponds to a maximum temporally entangled two-photon state. Thus, one can expect, in the same way that some quantum phenomena concerning spatial entanglement can be mimicked by spatially incoherent light, that some time-domain phenomena involving dispersion of temporal entanglement can be emulated by classical partially coherent light pulses, and vice versa.

\section{EXAMPLE: TEMPORAL GHOST INTERFERENCE WITH CLASSICAL PULSES}

Figure 1 shows a source that produces partially coherent light pulses described by $\Gamma_{e}$. Light is split into two arms. 


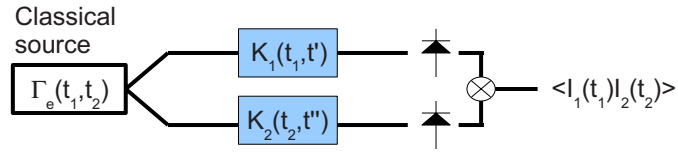

FIG. 1. (Color online) Schematic illustration of a fourth-order Hanbury Brown-Twiss-like time-domain interferometer with partially coherent classical-pulse input.

Each arm may be composed by cascading linear elements, such as temporal lenses, first-order dispersive elements, Gaussian filters, etc. [23]. Since we deal with linear propagation, in general the optical system in arm $\alpha$ can be mathematically characterized by the kernel [21] $K_{\alpha}\left(t, t^{\prime}\right), \alpha$ $=(1,2)$ (from now on we use $t$ to denote time in the retarded frame). At the end of the arm, we assume that a fast photodiode detects the radiation instantaneously. Then, the signal is further carried to a coincidence circuit that produces the average $\left\langle I_{1}\left(t_{1}\right) I_{2}\left(t_{2}\right)\right\rangle$, where $I_{\alpha}(t)$ is the instantaneous intensity arriving at detector from arm $\alpha$,

$$
I_{\alpha}(t)=\left|\int d t^{\prime} E_{\text {in }}\left(t^{\prime}\right) K_{\alpha}\left(t, t^{\prime}\right)\right|^{2} .
$$

Here $E_{\text {in }}(t)$ is the slowly varying envelope of a random realization of the input light. The average $\left\langle I_{1}\left(t_{1}\right) I_{2}\left(t_{2}\right)\right\rangle$ can be implemented with a time-to-amplitude converter followed by a multichannel analyzer. In [16], this operation is performed with a temporal resolution on the order of hundreds of picoseconds. It is important to note that we are dealing with the two-time intensity correlation function, not with an integrated version. Taking into account Eq. (5), and exchanging the order of integration and ensemble averaging, we obtain

$$
\begin{aligned}
\left\langle I_{1}\left(t_{1}\right) I_{2}\left(t_{2}\right)\right\rangle= & \int d t_{1}^{\prime} d t_{2}^{\prime} d t_{1}^{\prime \prime} d t_{2}^{\prime \prime}\left\langle E_{\mathrm{in}}^{*}\left(t_{1}^{\prime}\right) E_{\mathrm{in}}^{*}\left(t_{1}^{\prime \prime}\right) E_{\mathrm{in}}\left(t_{2}^{\prime}\right)\right. \\
& \left.\times E_{\mathrm{in}}\left(t_{2}^{\prime \prime}\right)\right\rangle K_{1}^{*}\left(t_{1}, t_{1}^{\prime}\right) K_{2}^{*}\left(t_{2}, t_{1}^{\prime \prime}\right) K_{1}\left(t_{1}, t_{2}^{\prime}\right) K_{2}\left(t_{2}, t_{2}^{\prime \prime}\right) .
\end{aligned}
$$

This fourth-order correlation can further be simplified by assuming the random input light to obey Gaussian statistics [18]. In this way, $\left\langle E_{\text {in }}^{*}\left(t_{1}^{\prime}\right) E_{\text {in }}^{*}\left(t_{1}^{\prime \prime}\right) E_{\text {in }}\left(t_{2}^{\prime}\right) E_{\text {in }}\left(t_{2}^{\prime \prime}\right)\right\rangle$ $=\Gamma_{e}\left(t_{1}^{\prime}, t_{2}^{\prime}\right) \Gamma_{e}\left(t_{1}^{\prime \prime}, t_{2}^{\prime \prime}\right)+\Gamma_{e}\left(t_{1}^{\prime}, t_{2}^{\prime \prime}\right) \Gamma_{e}\left(t_{1}^{\prime \prime}, t_{2}^{\prime}\right), \quad$ where $\quad \Gamma_{e}\left(t_{1}, t_{2}\right)$ $=\left\langle E_{\mathrm{in}}^{*}\left(t_{1}\right) E_{\mathrm{in}}\left(t_{2}\right)\right\rangle$. Then, we find that

$$
\left\langle I_{1}\left(t_{1}\right) I_{2}\left(t_{2}\right)\right\rangle=\left\langle I_{1}\left(t_{1}\right)\right\rangle\left\langle I_{2}\left(t_{2}\right)\right\rangle+\left|G^{(2)}\left(t_{1}, t_{2}\right)\right|^{2},
$$

where

$$
G^{(2)}\left(t_{1}, t_{2}\right)=\int d t_{1}^{\prime} d t_{2}^{\prime} \Gamma_{e}\left(t_{1}^{\prime}, t_{2}^{\prime}\right) K_{1}^{*}\left(t_{1}, t_{1}^{\prime}\right) K_{2}\left(t_{2}, t_{2}^{\prime}\right) .
$$

Clearly, $\left|G^{(2)}\right|^{2}$ is responsible for the interference effect. Our result in Eq. (7) is due to the Hanbury Brown-Twiss interferometer [18] in Fig. 1, operating with nonstationary light pulses. In a practical configuration, the background terms $\left\langle I_{\alpha}(t)\right\rangle$ can be independently measured, e.g., with a sufficiently fast oscilloscope, and then subtracted from Eq. (7). In analogy to the spatial case, $\left|G^{(2)}\right|^{2}$ plays the role of twophoton coincidence probability. If the background term is included, the maximum achievable visibility is $50 \%$. This is

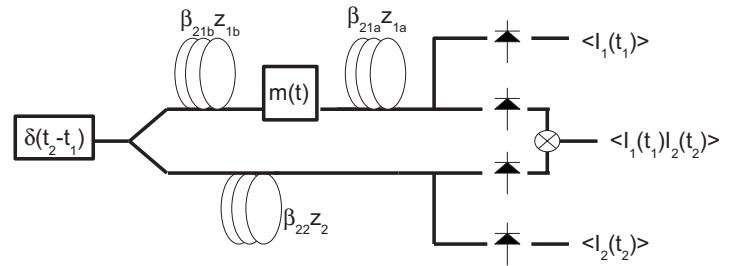

FIG. 2. Schematic view of a fourth-order interferometer, in which the arms contain first-order linear dispersive elements (single-mode fibers in the figure). Light from a temporally incoherent source obeying Gaussian statistics propagates in the device. A temporal modulator providing a deterministic gate $m(t)$ is placed in arm 1 . The average intensity in arms 1 and 2 before the detector is reached can be measured and subtracted from the coincidence signal. In this case the fourth-order interference has the same structure as if the input were temporally entangled two-photon light and time-domain ghost imaging of the gate is realized.

in clear distinction with the quantum case, where, in the small-photon-number regime, the visibility approaches the theoretical limit of $100 \%$ [10]. We emphasize that $G^{(2)}$ does not satisfy Eqs. (4), only $\Gamma_{e}$ does. However, the Gaussian statistics allow one to rewrite the fourth-order correlation in terms of second-order correlations. Only in this case do the analogies in fourth-order interferometry between the quantum and classical domains emerge $[13,14]$. We note also the similarity of Eq. (7) with the corresponding result for classical fourth-order interference in a spatial ghost diffraction scheme [24].

Let us now apply the scheme in Fig. 1 to the particular case of Fig. 2. In arm 1, we place a temporal modulator providing a deterministic gate $m(t)$. Before and after it we have two single-mode fibers (SMFs) with $\beta_{21 b}, \beta_{21 a}, z_{21 b}$, and $z_{21 a}$ the corresponding GVD coefficients and fiber lengths. In arm 2, we place another SMF characterized by $\beta_{22}$ and $z_{2}$. In this way [21]

$$
\begin{aligned}
K_{1}\left(t, t^{\prime}\right)= & (2 \pi i)^{-1}\left(\beta_{21 a} z_{21 a} \beta_{21 b} z_{21 b}\right)^{-1 / 2} \\
& \times \int d t^{\prime \prime} m\left(t^{\prime \prime}\right) \exp \left[-i \frac{\left(t^{\prime \prime}-t^{\prime}\right)^{2}}{2 \beta_{21 b} z_{1 b}}-i \frac{\left(t^{\prime \prime}-t\right)^{2}}{2 \beta_{21 a} z_{1 a}}\right],
\end{aligned}
$$

while

$$
K_{2}\left(t, t^{\prime}\right)=\left(2 \pi i \beta_{22} z_{2}\right)^{-1 / 2} \exp \left[-i \frac{\left(t-t^{\prime}\right)^{2}}{2 \beta_{22} z_{2}}\right] .
$$

The source is temporally incoherent and can be implemented by amplified spontaneous emission (ASE). In practice, the condition of temporal incoherence is satisfied when the coherence time of the ASE source is short enough compared with the photodiode resolution and the pulse width of the temporal window of $|m(t)|^{2}$. We then have $\Gamma_{e}\left(t_{1}, t_{2}\right) \propto \delta\left(t_{2}\right.$ $\left.-t_{1}\right)$. By substituting these equations into Eq. (8) and further selecting the fibers to match $\beta_{22} z_{2}=\beta_{21 b} z_{1 b}+\beta_{21 a} z_{1 a}$, we find that 


$$
\left|G^{(2)}\left(t_{2}-t_{1}\right)\right|^{2} \propto\left|M\left(\frac{t_{2}-t_{1}}{\beta_{21 a} z_{1 a}}\right)\right|^{2},
$$

where $M(\omega)$ is the Fourier transform of $m(t)$. In close analogy with the spatial case $[4,9,24]$, this situation thus leads to the temporal Fraunhofer pattern of the gate function. Here the temporal gate function corresponds to the object.

It is instructive to compare this result with the spectral domain experiments performed in [15] with a temporally entangled source. There, spectral filters are placed in one arm and this leads to spectral correlations when the measurements are made in coincidence. As expected, our temporally incoherent light source mimics the quantum entangled twophoton source. This constitutes the temporal counterpart phenomenon of ghost diffraction with partially coherent classical pulses. We note that the dispersion of the fiber naturally scales the shape of the Fourier transform.

\section{CONCLUSIONS}

In summary, we have demonstrated fourth-order temporal nonlocal interference with classical pulses assuming Gaussian statistics. The situation is identical with the ghost diffraction phenomena in the spatial domain. We have further sup- ported the connection between the quantum and classical, as well as temporal and spatial, domains by recalling that the equations that govern the spreading of temporal entanglement of the two-photon probability amplitude are identical with those describing the stretching of partially coherent light pulses in linearly dispersive media. This raises the question of whether classical, partially coherent light pulses may emulate the effects of time-domain quantum entanglement in fourth-order correlation experiments. The answer is expected to be important in view of the variety of applications that temporal entanglement currently offers in quantuminformation science. Our work is the first step toward addressing classical ghost diffraction phenomena in the temporal domain.

\section{ACKNOWLEDGMENTS}

This work was supported by Dirección General de Investigación Cientifica y Técnica, Spain, and FEDER, under Projects No. FIS2007-62217 and No. CONSOLIDER CSD 2007-013. V.T. acknowledges an FPU grant of the MEC. A.T.F. acknowledges funding from the Swedish Foundation for Strategic Research (SSF) and from the Academy of Finland.
[1] Y. H. Shih, Rep. Prog. Phys. 66, 1009 (2003).

[2] A. K. Ekert, J. G. Rarity, P. R. Tapster, and G. Massimo Palma, Phys. Rev. Lett. 69, 1293 (1992).

[3] For a recent review see M. D'Angelo and Y. H. Shih, Laser Phys. Lett. 2, 567 (2005).

[4] T. B. Pittman, Y. H. Shih, D. V. Strekalov, and A. V. Sergienko, Phys. Rev. A 52, R3429 (1995).

[5] D. Bouwmeester, J.-W. Pan, K. Mattle, M. Eibl, H. Weinfurter, and A. Zeilinger, Nature (London) 390, 575 (1997).

[6] A. N. Boto, P. Kok, D. S. Abrams, S. L. Braunstein, C. P. Williams, and J. P. Dowling, Phys. Rev. Lett. 85, 2733 (2000); M. D'Angelo, M. V. Chekhova, and Y. H. Shih, ibid. 87, 013602 (2001).

[7] M. B. Nasr, B. E. A. Saleh, A. V. Sergienko, and M. C. Teich, Phys. Rev. Lett. 91, 083601 (2003).

[8] D. V. Strekalov, A. V. Sergienko, D. N. Klyshko, and Y. H. Shih, Phys. Rev. Lett. 74, 3600 (1995).

[9] R. S. Bennink, S. J. Bentley, and R. W. Boyd, Phys. Rev. Lett. 89, 113601 (2002).

[10] A. Gatti, E. Brambilla, M. Bache, and L. A. Lugiato, Phys. Rev. Lett. 93, 093602 (2004); Phys. Rev. A 70, 013802 (2004).

[11] F. Ferri, D. Magatti, A. Gatti, M. Bache, E. Brambilla, and L. A. Lugiato, Phys. Rev. Lett. 94, 183602 (2005); M. Bache, D. Magatti, F. Ferri, A. Gatti, E. Brambilla, and L. A. Lugiato, Phys. Rev. A 73, 053802 (2006).

[12] For a recent review see A. Gatti, M. Bache, D. Magatti, E.
Brambilla, F. Ferri, and L. A. Lugiato, J. Mod. Opt. 53, 739 (2006).

[13] B. E. A. Saleh, A. F. Abouraddy, A. V. Sergienko, and M. C. Teich, Phys. Rev. A 62, 043816 (2000).

[14] B. E. A. Saleh, M. C. Teich, and A. V. Sergienko, Phys. Rev. Lett. 94, 223601 (2005).

[15] M. Bellini, F. Marin, S. Viciani, A. Zavatta, and F. T. Arecchi, Phys. Rev. Lett. 90, 043602 (2003); S. Viciani, A. Zavatta, and M. Bellini, Phys. Rev. A 69, 053801 (2004).

[16] A. Valencia, M. V. Chekhova, A. Trifonov, and Y. H. Shih, Phys. Rev. Lett. 88, 183601 (2002).

[17] M. Tsang and D. Psaltis, Phys. Rev. A 73, 013822 (2006).

[18] L. Mandel and E. Wolf, Optical Coherence and Quantum Optics (Cambridge University Press, Cambridge, U.K., 1995).

[19] H. Lajunen, P. Vahimaa, and J. Tervo, J. Opt. Soc. Am. A 22, 1536 (2005).

[20] S. A. Akhmanov, A. S. Chirkin, K. N. Drabovich, A. I. Kovrigin, R. V. Khokhlov, and A. P. Sukhorukov, IEEE J. Quantum Electron. 4, 598 (1968).

[21] B. E. A. Saleh and M. C. Teich, Fundamentals of Photonics (Wiley, New York, 1991); G. P. Agrawal, Nonlinear Fiber Optics (Academic Press, San Diego, CA, 2001).

[22] J. Lancis, V. Torres-Company, E. Silvestre, and P. Andrés, Opt. Lett. 30, 2973 (2005).

[23] M. Nakazawa, H. Kubota, and K. Tamura, IEEE J. Quantum Electron. 34, 1075 (1998).

[24] J. Cheng and S. Han, Phys. Rev. Lett. 92, 093903 (2004). 